Proc. 13th Econophysics Colloquium (EC) and 9th Symposium of Physics in Economy and Social Sciences (FENS), 2017

\title{
Influence of a Range of Interaction among Agents on Efficiency and Effectiveness of Knowledge Transfer within an Organisation
}

\author{
K. PARAdowski ${ }^{a}$, A. Kowalska-STYCZEN ${ }^{b, *}$ And K. MAlarz ${ }^{a}$ \\ ${ }^{a}$ AGH University of Science and Technology, Faculty of Physics and Applied Computer Science, \\ Al. Mickiewicza 30, 30-059 Krakow, Poland \\ ${ }^{b}$ Silesian University of Technology, Faculty of Organisation and Management, \\ T. Roosevelta 26/28, 41-800 Zabrze, Poland
}

\begin{abstract}
In this study we examined how the size of non-formal groups between organisation members affect the transfer of knowledge in the context of the efficiency and effectiveness of this process. To analyse the dynamics of the transfer of knowledge the cellular automata model was used. The model is based on local interactions between members of the organisation, that take place in the nearest neighbourhood. These groups of close neighbours are represented by von Neumann's neighbourhood (four nearest-neighbours) and Moore's neighbourhood (four nearest-neighbours and four next-nearest neighbours) and complex neighbourhood (four nearest neighbours, four next-nearest neighbours and four next-next-neighbours). The results of the simulation show the influence of the size of the neighbourhood on the efficiency of knowledge transfer.
\end{abstract}

DOI: 10.12693 /APhysPolA.133.1470

PACS/topics: cellular automata, complex systems, social and economic systems, structures and organisation in complex systems

\section{Introduction}

Today in a fast-changing environment, knowledge is the dominant source of competitive advantage [1-3]. In literature, there are many definitions of knowledge. For example, Applehans et al. [4] define knowledge as information used to solve a problem. Davenport and Prusak [5] point out, that knowledge is a characteristics of human mind and it is an inherent part of human complexity and unpredictability. This is also confirmed by the Buckman of Bucman Labs study, which shows that $90 \%$ of the knowledge in each organisation is contained in "people's heads" [6]. The core process in an organisation, where knowledge is present is a knowledge transfer. Knowledge transfer in an organisation is the process through which one person or group influences the experience of others [7]. Knowledge is created when people communicate and share knowledge, assimilate and apply what they have learned. As knowledge transfer is essential for many organisational processes, including best practice transfer, product development and organisational survival [8], its effectiveness and efficiency are particularly important.

Knowledge and its distribution are strongly linked to social interactions. Managers receive two-thirds of information and knowledge through face-to-face communication or telephone conversations, and only one-third

\footnotetext{
* corresponding author; e-mail:

Agnieszka.Kowalska-Styczen@polsl.pl
}

come from documents [5]. The importance of social interaction, especially informal contacts in the knowledge transfer process, has been demonstrated by many authors [3, 8-11]. Therefore, in this article, knowledge transfer is understood as a common creative process in an organisation, which is most often done informally, by sharing face-to-face knowledge, as in Ref. [12].

Our goal is simulation and bottom-up approaches in modelling the transfer of knowledge, where local (bottom-up) relationships generate phenomena at a global level (that is, at the level of the whole organisation). The basic premise of the model, inspired by Reagans and McEvily [8], is to divide the knowledge transferred into a number of portions (chunks) of knowledge. Informal contacts between members of the organisation are represented in our research by different neighbourhoods size (four, eight or twelve elements), because groups of employees consist of a different number of members.

The results presented here base on model of knowledge transfer within a small or a medium organisation [13], where agents send and recipe chunks of knowledge only when distances (i) in space among agents (ii) and in knowledge are small. Removing the latter restriction leads to more efficient and more effective knowledge transfer [14].

Here we would like to check if relaxing the spatial restriction also may be helpful in spreading knowledge among agents in artificial organisation. Similarly to our earlier approaches $[13,14]$ we will base our discussion of the results on the computer simulations based on cellular automata $(\mathrm{CA})$ technique. 


\section{Model}

To define CA $[15,16]$ one should specify (i) a regular grid $\mathcal{G}$ of sites $\xi$, (ii) a set $\mathcal{S}=\left\{s_{1}, \cdots, s_{N}\right\}$ of available sites states, (iii) and a rule $\mathcal{F}$ governing the time evolution of the system. The latter defines a state $s(\xi ; t+1)$ of site $\xi \in \mathcal{G}$ at time $t+1$ basing of the states of site $\xi$ neighbourhood $\mathcal{N}$ at time $t$ :

$$
s(\xi ; t+1)=\mathcal{F}\left(s(\xi ; t), s\left(\xi_{1} ; t\right), \cdots, s\left(\xi_{M} ; t\right)\right)
$$

and $\xi_{i=1, \ldots, M} \in \mathcal{N}^{\prime}=\mathcal{N} \backslash\{\xi\}$, where $M$ is a number of sites in deleted neighbourhood $\mathcal{N}^{\prime}$ of $\xi$.

\subsection{Set $\mathcal{S}$}

Similarly to our earlier approaches $[13,14]$ every agent is characterised by a Boolean vector variable

$$
\boldsymbol{C}(\xi ; t)=\left[c_{1}(\xi ; t), c_{2}(\xi ; t), \ldots, c_{K}(\xi, t)\right],
$$

where $c_{i}(\xi ; t) \in\{0,1\}$ describes lack $\left[c_{i}(\xi ; t)=0\right]$ or possessing $\left[c_{i}(\xi ; t)=1\right] i$-th chunk of knowledge by the agent at site $\xi$ and at time $t$. $K$ stands for the number of all chunks of knowledge available for every agent.

\subsection{Rule $\mathcal{F}$}

The rule $\mathcal{F}$ states, that during each simulation step $t$ each agent may receive a single chunk of knowledge from the randomly selected agent in his/her deleted neighbourhood $\mathcal{N}^{\prime}$. However, the sender of this information (placed at position $\xi^{\prime} \in \mathcal{N}^{\prime}$ ) is willing to share his/her knowledge only when the recipient is smart enough. Namely, the chunk of knowledge is transferred from sender (at site $\xi^{\prime}$ ) to recipient (at site $\xi$ ) only when the difference in number of possessed chunk of knowledge among these two agents is exactly equal to one

$$
\wedge\left[\sum_{j=1}^{K} c_{j}\left(\xi^{\prime} ; t\right)-\sum_{j=1}^{K} c_{j}(\xi ; t)\right]=1 .
$$$$
c_{i}(\xi ; t+1)=1 \Longleftrightarrow c_{i}(\xi ; t)=0 \wedge c_{i}\left(\xi^{\prime} ; t\right)=1
$$

Such approach is not far from Deffuant et al. [17] model of opinion dynamics, where opinion exchange among agents is possible only when sender and recipient have similar opinions [18-22] and consistent with empirical findings regarding knowledge transfer in organisation [8]. Also simulations driven by the homophily principle assume that "agents are likely to exhibit strong preferences towards agents with which they are similar" [23]. Our approach is close in spirit to Axelrod model of dissemination of culture [24]. The main difference is that in our model, the current of influence is unidirectional.

\subsection{Grid $\mathcal{G}$}

We assume that agents occupy the nodes of a square lattice with linear size $L$ :

$$
\mathcal{G}=\{(x, y): x, y \in \mathbb{N} \wedge 1 \leq x, y \leq L\} .
$$

Additionally, the periodic boundary conditions are assumed.
In order to check the influence of the range of interaction on efficiency and effectiveness of knowledge transfer we consider three kinds of neighbourhoods $\mathcal{N}$. The site $\xi \in \mathcal{G}$ and its $M=4$ nearest-neighbours constitute the von Neumann neighbourhood

$$
\mathcal{V}=\{(x, y),(x, y \pm 1),(x \pm 1, y)\},
$$

while for the Moore neighbourhood also four next-nearest neighbours are included $(M=8)$ :

$$
\mathcal{M}=\mathcal{V} \cup\{(x-1, y \pm 1),(x+1, y \pm 1)\} .
$$

Finally, we apply a complex neighbourhood with nextnext-nearest neighbours $(M=12)$ :

$$
\mathcal{C}=\mathcal{M} \cup\{(x, y \pm 2),(x \pm 2, y)\} .
$$

\section{Results}

We measure the efficiency of the knowledge transfer as a time $\tau$ necessary for reaching the steady state of the system. Daft ([25], p. 663) defines effectiveness as "the degree to which goals are attained' and efficiency as 'amount of resources used to produce a unit of output". In order to evaluate the effectiveness of the knowledge transfer we study the average coverage of chunks of knowledge in the system $\langle f\rangle$ and the fraction $n(K)$ of agent having all $(K)$ chunks of knowledge which are available in the system.

Qualitatively, the results are not surprising: both effectiveness and efficiency increase with the neighbourhood radius. Numerical calculations reveal some nontrivial quantitative details which deserve attention.

In Fig. 1 the time evolution of the fraction $n(k)$ of agents having $k$ chunks of knowledge for $L=20$ and various (i) initial concentration of chunks of knowledge $(p=0.2,0.5,0.8)$ and (ii) various values of $K$ are presented. The results are obtained for von Neumann (a-c, $\mathrm{j}-\mathrm{l})$, Moore (d-f, m-o), and complex (g-i, p-r) neighbourhood. The values presented in Figs. 1-5 are averaged over $R=10^{4}$ independent simulations. As we can see, the shape of neighbourhood does not influence the time evolution of $n(k)$ too much. The system is much more vulnerable to the changes of initial concentration of chunks of knowledge $p$ [13]. However - particularly for larger $K$ and larger $p$ - we can see that fraction of $n(k=K)$ grows slightly with the number $M$ of sites constituting the neighbourhood.

\subsection{Effectiveness of the knowledge transfer}

In Fig. 2 the time evolution of the fraction $n(K)$ of agents having all available $(K)$ chunks of knowledge for $L=20, K=4$ and various initial level of knowledge in organisation $p$ are presented. Again, the shape of neighbourhood does not affect the level on which fraction $n(K)$ saturates, however, the time of reaching the stationary state is reduced roughly twice when we change the von Neumann neighbourhood to the Moore one. This may suggest that kind of neighbourhood may have greater impact on efficiency than on effectiveness of knowledge transfer. 
(a) $\mathcal{V}: K=4, p=0.2$

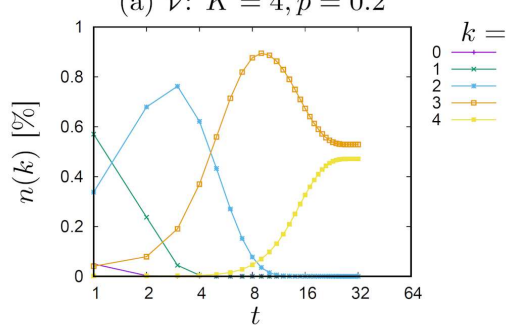

(d) $\mathcal{M}: K=4, p=0.2$

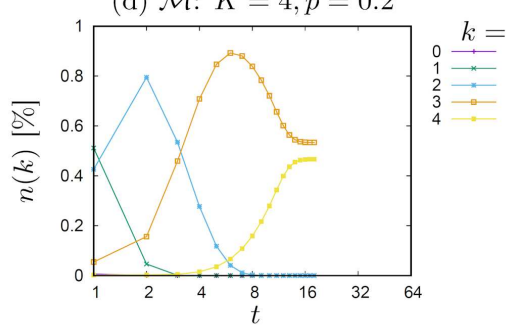

(g) $\mathcal{C}: K=4, p=0.2$

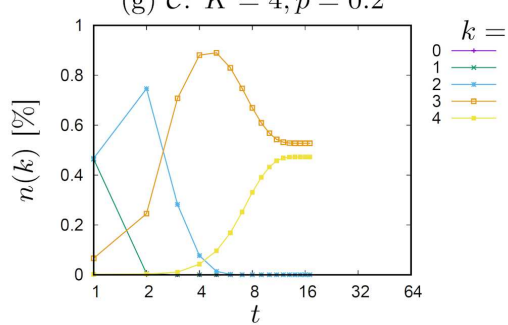

(j) $\mathcal{V}: K=8, p=0.2$

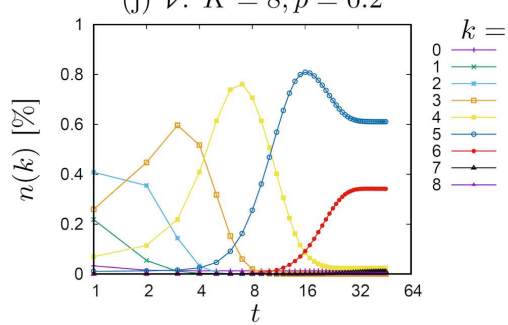

(m) $\mathcal{M}: K=8, p=0.2$

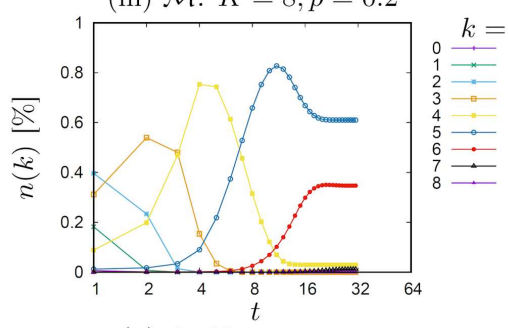

(p) $\mathcal{C}: K=8, p=0.2$

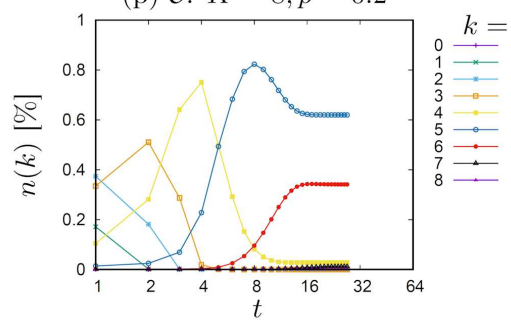

(b) $\mathcal{V}: K=4, p=0.5$

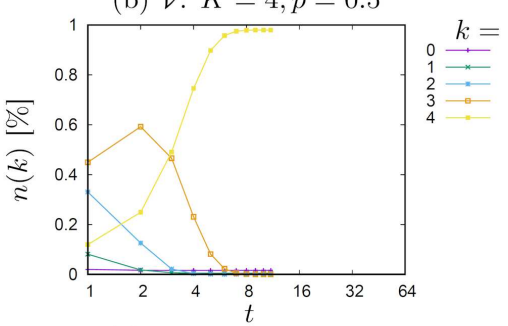

(e) $\mathcal{M}: K=4, p=0.5$

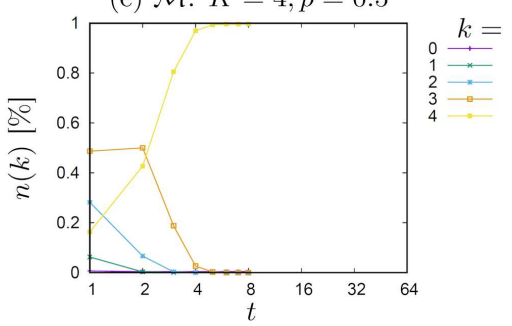

(h) $\mathcal{C}: K=4, p=0.5$

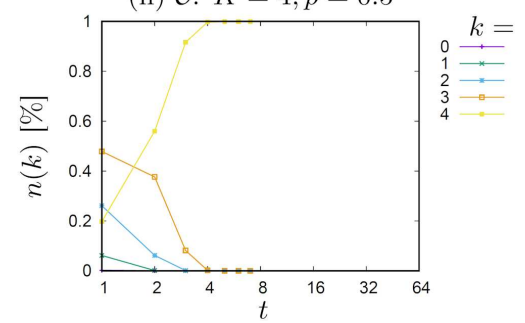

(k) $\mathcal{V}: K=8, p=0.5$

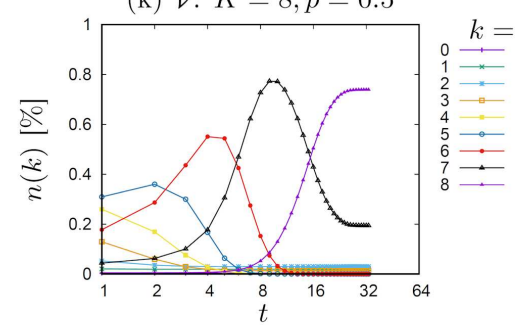

(n) $\mathcal{M}: K=8, p=0.5$

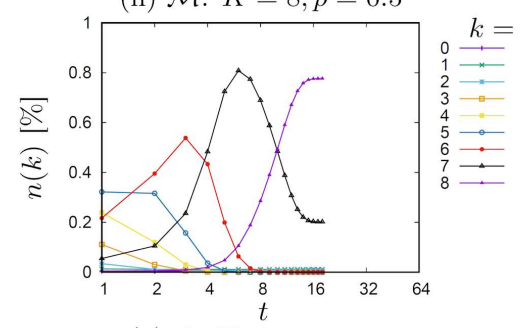

(q) $\mathcal{C}: K=8, p=0.5$

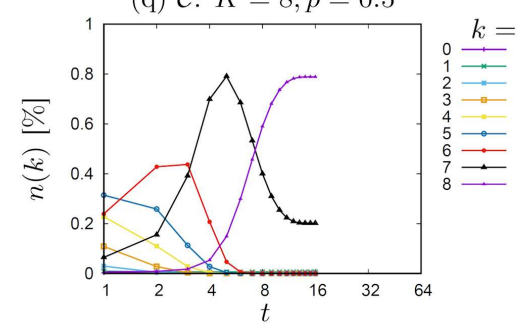

(c) $\mathcal{V}: K=4, p=0.8$

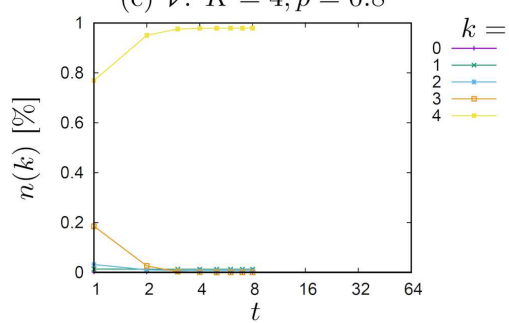

(f) $\mathcal{M}: K=4, p=0.8$

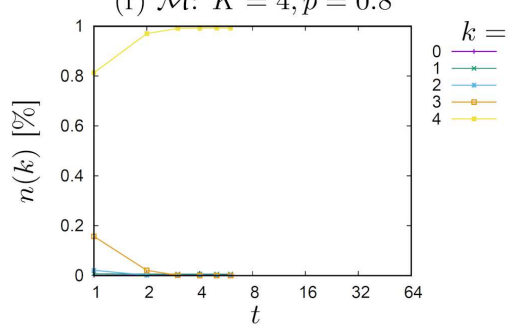

(i) $\mathcal{C}: K=4, p=0.8$

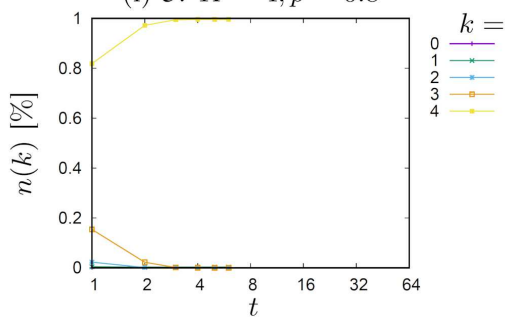

(1) $\mathcal{V}: K=8, p=0.8$

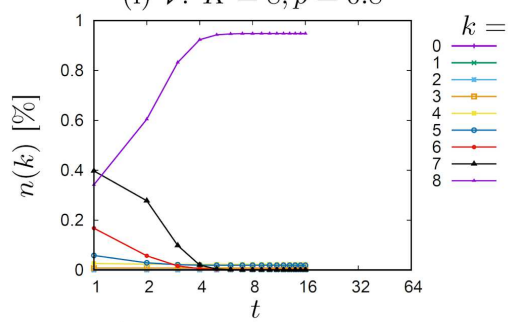

(о) $\mathcal{M}: K=8, p=0.8$

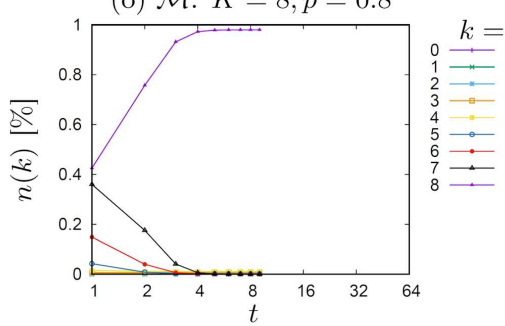

(r) $\mathcal{C}: K=8, p=0.8$

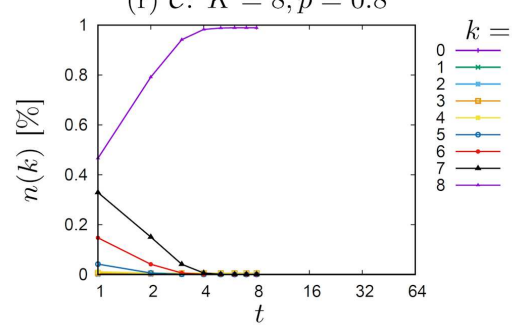

Fig. 1. The time evolution of the fraction $n(k)$ of agents having $k$ chunks of knowledge for $L=20$ and various (i) initial concentration of chunks of knowledge $(p=0.2,0.5,0.8)$ and (ii) various values of $K$. The results are obtained for von Neumann (a-c, j-l), Moore (d-f, m-o), and complex (g-i, p-r) neighbourhood. The values of $n(k)$ are averaged over $R=10^{4}$ independent simulations. 

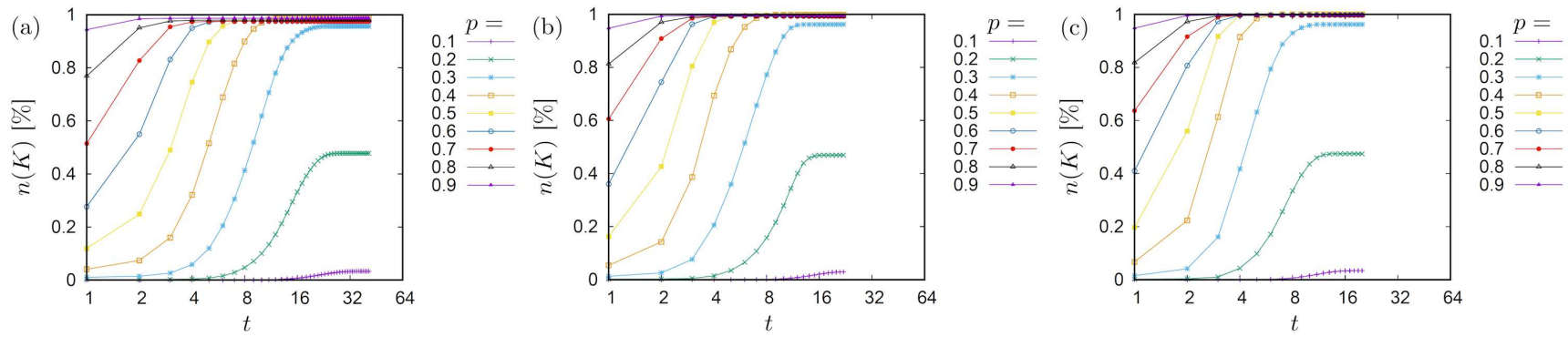

Fig. 2. The time evolution of the fraction $n(K)$ of agents having all available $(K)$ chunks of knowledge for $L=20$, $K=4$ and various initial level of knowledge in organisation $p$. The values of $n(K)$ are averaged over $R=10^{4}$ independent simulations. (a) von Neumann neighbourhood, (b) Moore neighbourhood, (c) complex neighbourhood.

(a) $\mathcal{V}: K=4$

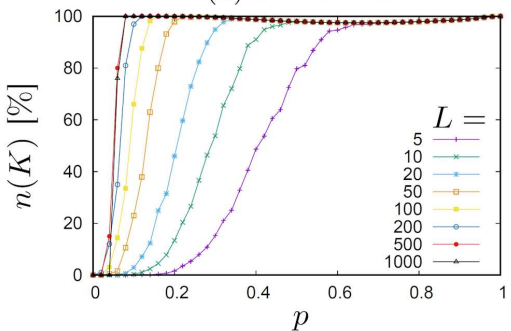

(d) $\mathcal{V}: K=4$

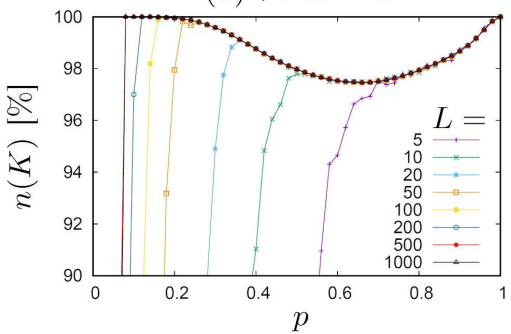

(g) $\mathcal{V}: K=8$

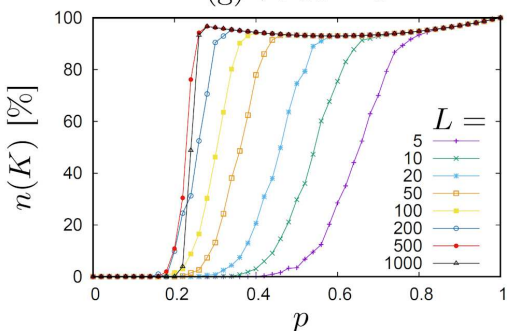

(j) $\mathcal{V}: K=8$

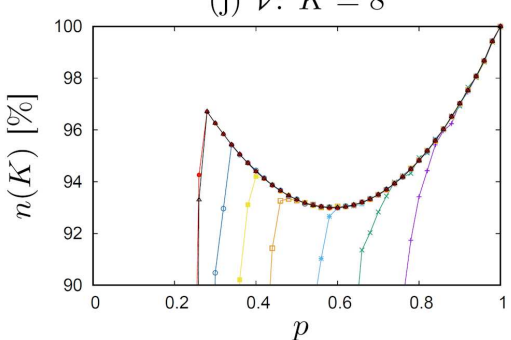

(b) $\mathcal{M}: K=4$

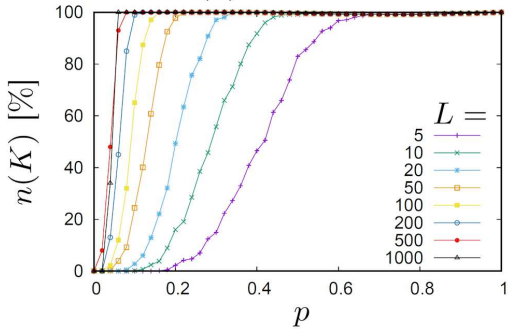

(e) $\mathcal{M}: K=4$

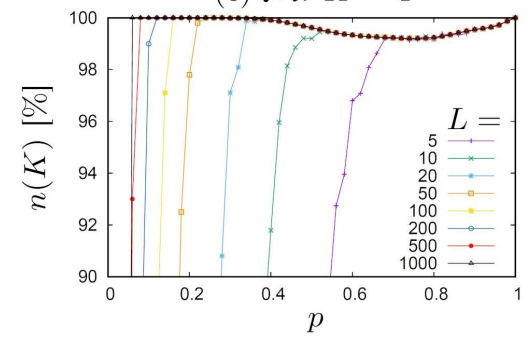

(h) $\mathcal{M}: K=8$

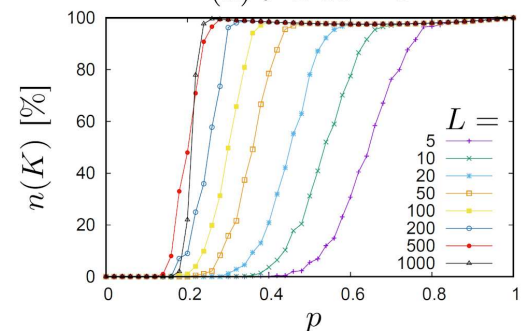

(k) $\mathcal{M}: K=8$

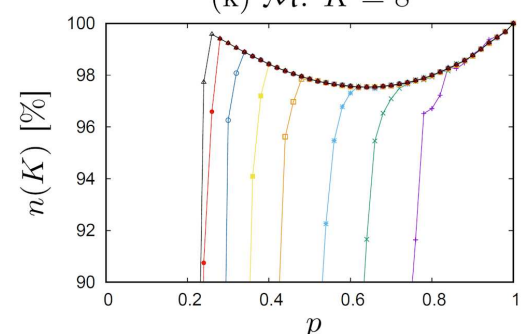

(c) $\mathcal{C}: K=4$

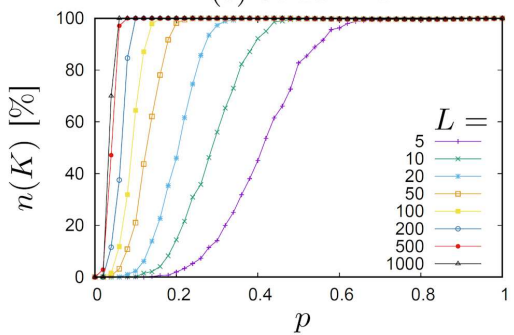

(f) $\mathcal{C}: K=4$

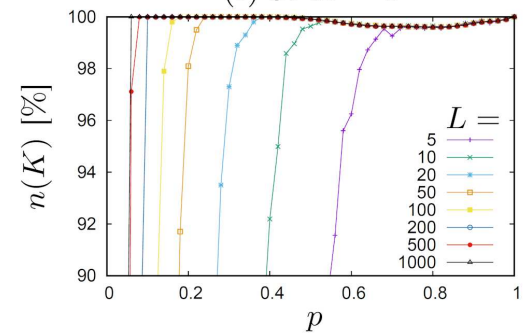

(i) $\mathcal{C}: K=8$

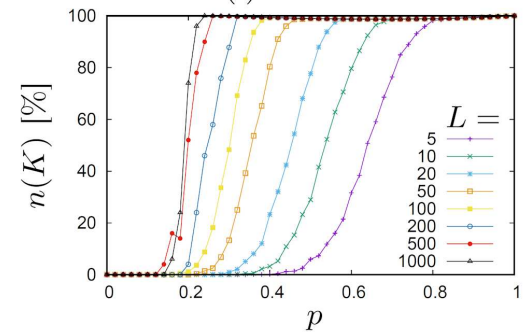

(l) $\mathcal{C}: K=8$

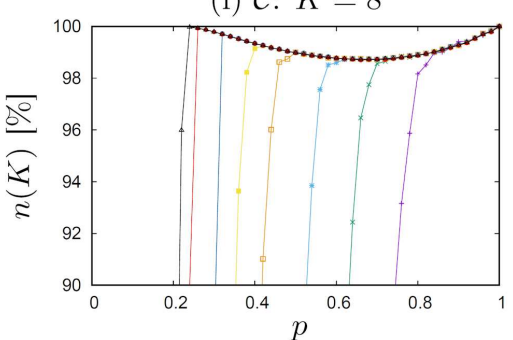

Fig. 3. The fraction of agents having total knowledge $\sum_{i=1}^{K} c_{i}=K=8$ vs. initial knowledge in the system $(p)$ for various system sizes $L$ and various neighbourhoods. The results are averaged over $R=10^{4}$ independent simulations.

In Fig. 3 the fraction of agents having total knowledge $\sum_{i=1}^{K} c_{i}=K$ vs. initial knowledge in the system $(p)$ for various system sizes $L$ and various neighbourhoods are presented. Figures $3 \mathrm{~d}-\mathrm{f}$ and $\mathrm{j}-\mathrm{l}$ present top $10 \%$ of 
Figs. 3a-c and g-i, respectively. For von Neumann neighbourhood and medium $(L=10)$ and larger organisations we observe minimum of $n(K)$ curves for $p \approx 0.6$ [13]. This counter-intuitive effect is directly associated with restriction (1b) - for high enough initial concentration of chunks of knowledge $p$ some agents acquire high level of competences quite quickly and do not want share their knowledge with their not-so-smart neighbours. The effect may be reduced [14] when agents receive chunks of knowledge from much smarter agents

$$
\left[\sum_{j=1}^{K} c_{j}\left(\xi^{\prime} ; t\right)-\sum_{j=1}^{K} c_{j}(\xi ; t)\right] \geq 1
$$

and even vanishes [14] when sender is smarter than or as smart as recipient of chunk of knowledge

$$
\left[\sum_{j=1}^{K} c_{j}\left(\xi^{\prime} ; t\right)-\sum_{j=1}^{K} c_{j}(\xi ; t)\right] \geq 0 .
$$

Also an increasing the range of interaction may help in reducing this effect. The reduction is stronger for com- plex neighbourhood (Fig. 3c, f, i, l) than for Moore's one (Fig. 3b, e, h, k).

In Fig. 4 the average coverage $\langle f\rangle$ of chunks of knowledge in organisation for small ( $L=5$, Figs. 4a, c, e) and medium ( $L=20$, Figs. 4 b, d, f) sizes of organisation and various values of $K$ and neighbourhoods are presented. Again, for $K<8$ one may observe non-monotonous dependence of the average coverage $\langle f\rangle$ of chunks of knowledge in organisation vs. initial concentration of chunks of knowledge $p$ [13]. The changes in knowledge transfer rules from Eq. (1b) to Eq. (2a) or Eq. (2b) generates a monotonous dependence $\langle f\rangle$ vs. $p$ [14]. Moreover, for rule Eq. (2b) we observe a collapse of curves for various values of $K$ to a single curve close to Heaviside's step function $\Theta(p-0.05)$. The changes in range of neighbourhoods do not yield so spectacular changes in shapes of $\langle f\rangle$ vs. $p$ curves. However, the reduction of barriers of knowledge transfer for all considered values of $K$ may be observed as shallowing minimum of $\langle f\rangle$ vs. $p$ dependences in interval $p \in[0.5,1]$. (a) $\mathcal{V}: L=5$

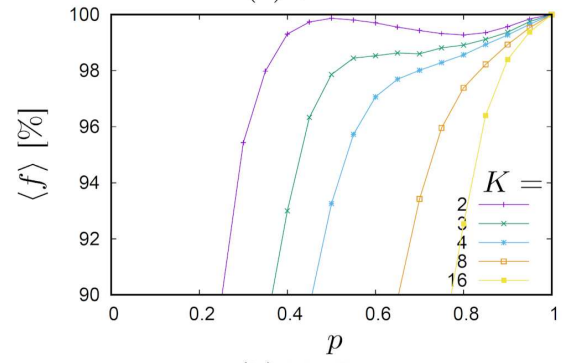

(b) $\mathcal{V}: L=20$

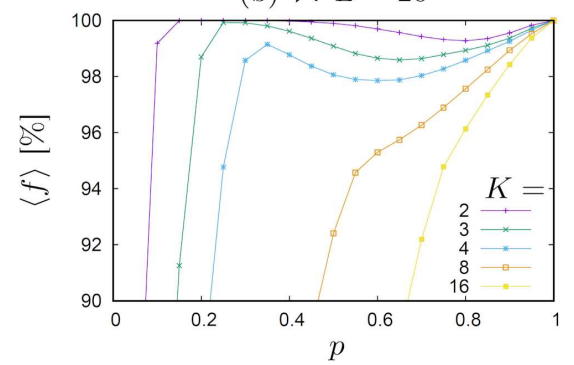

(c) $\mathcal{M}: L=5$

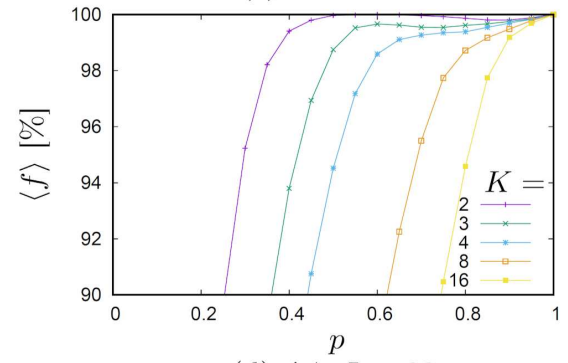

(d) $\mathcal{M}: L=20$

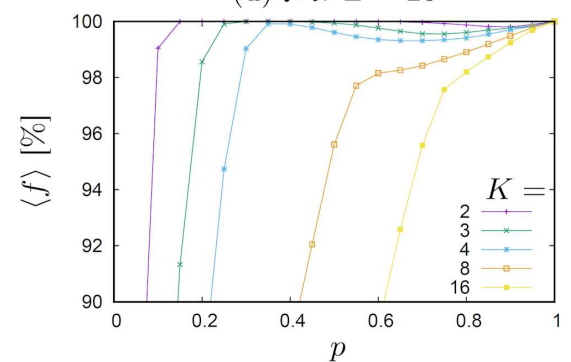

(e) $\mathcal{C}: L=5$

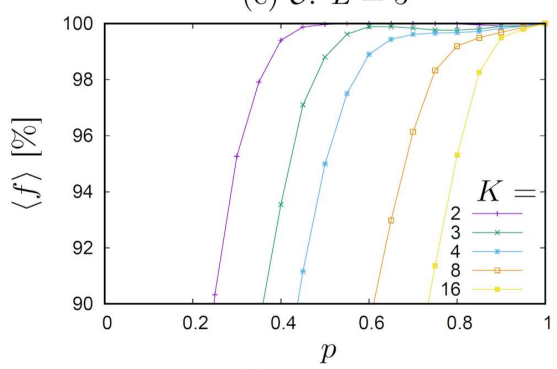

(f) $\mathcal{C}: L=20$

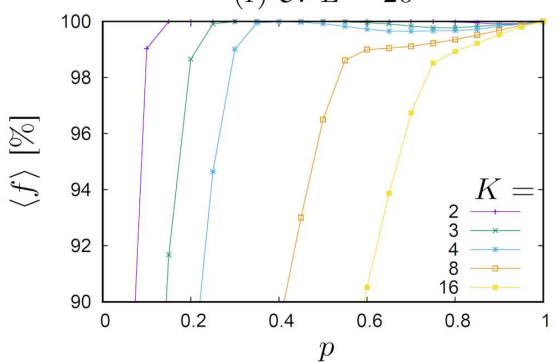

Fig. 4. The average coverage $\langle f\rangle$ of chunks of knowledge in organisation for small (a, c, e, $L=5)$ and average (b, d, $\mathrm{f}, L=20)$ size of organisation and various neighbourhoods. The values of $\langle f\rangle$ are averaged over $R=10^{4}$ independent simulations.

\subsection{Efficiency of the knowledge transfer}

In Fig. 5 the times $\tau$ necessary for reaching the stationary state of the system for (a) $K=4$ and (b) $K=8$ chunks of knowledge available in the system for $L=20$ are presented. The solid line curves show low degree polynomial fits as a guide for eyes. As we mentioned in Sect. 3.1 the number of sites in neighbourhood may influence the efficiency of knowledge transfer more than the effectiveness of this process. Indeed, the change of neighbourhood (for fixed $p$ ) may lead to reduction of time $\tau$ of reaching the stationary state of the system even twice. It is caused by the fact that in the larger neighbourhood there are more agents with whom knowledge transfer is possible.

Also the probability $p$ effects the transfer time $\tau$. We observe that transfer time increases with $p$ till $p \approx$ 0.2 and then decreases for larger $p$. This situation is generic for all types considered neighbourhoods, The nonmonotonic dependence of $\tau(p)$ and the position of its maximum may be directly connected with the shape of a probability function of knowledge transfer among agents, as presented in Fig. 9 in Ref. [13]. 
(a) $L=20, K=4$

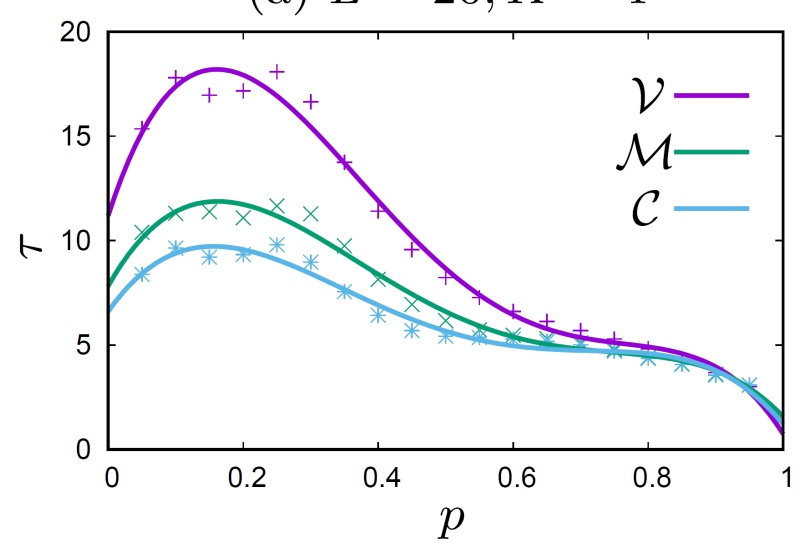

(b) $L=20, K=8$

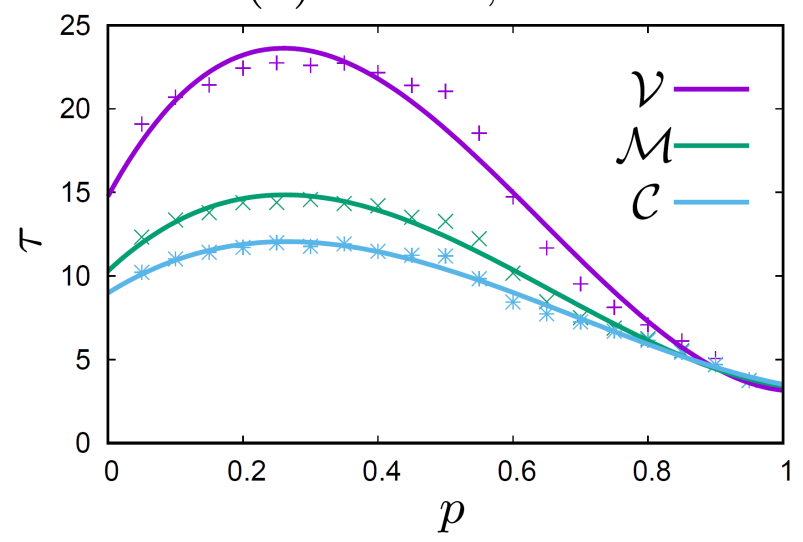

Fig. 5. The time $\tau$ necessary for reaching the stationary state of the system for (a) $K=4$ and (b) $K=8$ chunks of knowledge available in the system for $L=20$. The values of $\tau$ are averaged over $R=10^{4}$ independent simulations. The solid line curves show low degree polynomial fits.

The probability of knowledge transfer for low values of $p$ may be deduced from simplified mean-field-like approach based on formula for probability of meeting pair of agents possessing zero and only single chunk of knowledge (see Eq. (2) and Fig. 9 in Ref. [13] and discussion there). For low concentration of chunks of knowledge $p$, the probability of meeting a pair of agents with zero and single chunk of knowledge increases with $p$ till $p \approx 0.2$ and then decreases. This situation is not dissimilar with qualitative behaviour of $\tau(p)$ dependence.

The difference in $\tau(p)$ vanishes for larger values of initial concentration of chunks of knowledge in organisation $(p>0.8)$ as in this limit independently of kind of neighbourhood almost all agents acquire almost all chunks of knowledge in several time steps.

\section{Discussion and conclusions}

The proposed model has been designed to investigate the impact of the size of informal groups in the organisation on the effectiveness and efficiency of knowledge transfer. Three different neighbourhoods: the von
Neumann neighbourhood with four nearest-neighbours, the Moore neighbourhood with eight neighbours and the complex one with twelve neighbours have been adopted. Our results show that the size of the neighbourhood has a far greater impact on the efficiency than on the effectiveness of the knowledge transfer. As can be seen, the knowledge transfer time is shorter in the case of a larger neighbourhood. This may be related to the cohesion of the network of agents. We understand cohesion as Fleming et al. ([26] and references therein) i.e. "a cohesive social structure [take place when] most people have direct ties to each others in the network". Of course, the $\mathrm{CA}$ technique applied here does not allow for exact implementation of this definition in our case. Greater cohesion occurs when people have dense and overlapping relationships [26]. This situation of overlapping ties occurs in the Moore neighbourhood for eight neighbours and complex neighbourhood for twelve neighbours where each site belongs to neighbourhoods of several sites. If the lattice is fully occupied (i.e. it is not diluted, and every site is occupied by an agent), the agents have a greater number of common neighbours (and thus overlapping ties) than those of the von Neumann neighbourhood for four nearest-neighbours. In the context of social capital considerations, the overlapping relationships (a greater closure of the contact structure, when we have mutual friends who know each other and make contact with each other) raises greater trust between people and thus improves the flow of information [27]. This is also confirmed by the results of our simulation.

Of course, the results of the simulations encourage further research and exploration of factors that influence the transfer of knowledge, as well as the premise of empirical research on the impact of employee-to-employee networking on knowledge exchange. The model presented in this work can be easily extended and other factors describing the transfer of knowledge can be taken into account. One of them could be homophily because research show that it affects the frequency of the interaction between agents [23].

\section{Acknowledgments}

This research was supported by National Science Centre (NCN) in Poland (grant no. UMO2014/15/B/HS4/04433) and partially supported by AGH-UST statutory tasks No. 11.11.220.01/2 within subsidy of Ministry of Science and Higher Education.

\section{References}

[1] C. Chen, RESD Management 34, 311 (2004).

[2] M. Lyles, J. Salk, J. Int. Business Stud. 27, 877 (1996).

[3] W. Tsai, Acad. Manage. J. 44, 996 (2001).

[4] W. Applehans, A. Globe, G. Laugero, Managing Knowledge. A Practical Web-Based Approach, Addison-Wesley, Reading (MA) 1999. 
[5] T.H. Davenport, L. Prusak, Working Knowledge: How Organizations Manage What They Know, Harvard Business School Press, Boston (MA) 2000.

[6] L. Wah, Manage. Rev. 88, 17 (1999).

[7] L. Argote, P. Ingram, Organiz. Behav. Human Decis. Process. 82, 150 (2000).

[8] R. Reagans, B. McEvily, Administr. Sci. Quarterly 48, 240 (2003).

[9] P. Ingram, P. Roberts, Am. J. Sociol. 106, 387 (2000).

[10] R. Reagan, E.W. Zuckerman, Organiz. Sci. 12, 502 (2001)

[11] C. Chen, J. Huang, Int. J. Informat. Manage. 27, 104 (2007).

[12] L. Girdauskiene, A. Savaneviciene, Proced. - Social Behav. Sci. 41, 15 (2012).

[13] A. Kowalska-Styczen, K. Malarz, K. Paradowski, J. Artif. Societ. Social Simulat. 21, (2) 3 (2018).

[14] A. Kowalska-Styczen, K. Malarz, K. Paradowski, in: Proc. 10th Int. Conf. on Agents and Artificial Intelligence, Eds. A.P. Rocha, J. van den Herik, Scitepress, 2018, p. 151.

[15] S. Wolfram, A New Kind of Science, Wolfram Media, Champaign (IL) 2002
[16] A. Ilachinski, Cellular Automata: A Discrete Universe, World Sci., Singapore 2001.

[17] G. Deffuant, D. Neau, F. Amblard, G. Weisbuch, Adv. Complex Syst. 3, 87 (2000).

[18] R. Hegselmann, U. Krause, J. Artif. Societ. Social Simulat. 5, (3) 2 (2002).

[19] K. Malarz, Int. J. Mod. Phys. C 17, 1521 (2006).

[20] K. Kułakowski, Physica A Statist. Mech. Appl. 388, 469 (2009).

[21] K. Malarz, P. Gronek, K. Kułakowski, J. Artif. Societ. Social Simulat. 14, (1) 2 (2011).

[22] K. Malarz, K. Kułakowski, Europhys. News 45, 21 (2014).

[23] B.R. Hirshman, J.S. Charles, K.M. Carley, Computat. Math. Organiz. Theory 17, 318 (2011).

[24] R. Axelrod, J. Conflict Resol. 41, 203 (1997).

[25] R.L. Daft, Organization Theory and Design, SouthWestern College Publ., Cincinnati (OH) 1998.

[26] L. Fleming, S. Mingo, D. Chen, Adminis trat. Sci. Quarterly 52, 443 (2007).

[27] J.S. Coleman, Am. J. Sociol. 94, S95 (1988). 